\title{
Cardiac magnetic resonance visualizes acute and chronic myocardial injuries in myocarditis
}

\author{
Knut Haakon Stensaeth • Pavel Hoffmann • \\ Eigil Fossum • Arild Mangschau • Leiv Sandvik • \\ Nils Einar Klow
}

Received: 5 October 2010/Accepted: 17 January 2011/Published online: 24 February 2011

(C) The Author(s) 2011. This article is published with open access at Springerlink.com

\begin{abstract}
Our objective was to evaluate the ability of CMR to visualize myocardial injuries over the course of myocarditis. We studied 42 patients (39 males, 3 females; age $37 \pm 14$ years) with myocarditis during the acute phase and after $12 \pm 9$ months. CMR included function analyses, T2-weighted imaging (T2 ratio), T1-weighted imaging before and after i.v. gadolinium injection (global relative enhancement; gRE), and late gadolinium enhancement (LGE). In the acute phase, the T2 ratio was elevated in $57 \%$, gRE in $31 \%$, and LGE was present in $64 \%$ of the patients. In 32 patients $(76 \%)$ were any two (or more) out of three sequences abnormal. At follow-up, there was an increase in ejection fraction $(57.4 \pm$ $11.9 \%$ vs. $61.4 \pm 7.6 ; P<0.05)$ while both $\mathrm{T} 2$ ratio $(2.04 \pm 0.32$ vs. $1.70 \pm 0.28 ; P<0.001)$ and $\mathrm{gRE}$ $(4.07 \pm 1.63$ vs. $3.11 \pm 1.22 ; P<0.05)$ significantly
\end{abstract}

K. H. Stensaeth · P. Hoffmann - E. Fossum - N. E. Klow Department of Radiology, Oslo University Hospital, Ullevaal, Oslo, Norway
A. Mangschau
Department of Cardiology, Oslo University Hospital,
Ullevaal, Oslo, Norway
L. Sandvik
Centre for Clinical Research, Oslo University Hospital, Ullevaal, Oslo, Norway
K. H. Stensaeth $(\bowtie)$
Kirkevn. 166, 0407 Oslo, Norway
e-mail: k.h.stensath@medisin.uio.no

decreased. The LGE persisted in 10 patients. Dilated cardiomyopathy was present in 3 patients and 4 patients received a defibrillator or a pacemaker. A comprehensive CMR approach is a useful tool to visualize myocardial tissue injuries over the course of myocarditis. CMR may help to differentiate acute from healed myocarditis, and add information for the differential diagnoses.

Keywords Myocarditis - Coronary angiography . Cardiac magnetic resonance $\cdot$ Myocardial injury · Differential diagnosis

\section{Introduction}

The myocardium can be affected by a wide spectrum of tissue injuries [1]. Non-invasive differentiation of these injuries might be clinically important. Myocarditis has been reported in up to $12 \%$ of young adults presenting with sudden death [2], and is an important underlying aetiology of other myocardial diseases such as dilated [3] and arrhytmogenic right ventricular [4] cardiomyopathy.

In patients with a clinical history, symptoms, and physical findings that suggest acute myocardial disease, a combination of electrocardiogram (ECG) and seromarkers such as troponin is used to determine acuity, extent, and location of the myocardial injury. However, current diagnostic approaches have 
limitations. The accuracy of ECGs to detect myocardial ischemia is often low, which may result in inappropriate treatment [5]. The diagnostic accuracy of these diagnostic tools is even lower when applied to patients with myocarditis [6]. Endomyocardial biopsy (EMB), though the gold standard for tissue pathology, does not have a clinical role in acute myocardial diseases, and clinical indications in acute myocarditis are limited to patients with otherwise unexplained heart failure [7]. Also, it is well known that myocardial injuries such as oedema or capillary leakage are difficult to assess histopathologically.

It is therefore important to establish the pattern and reversibility of injuries in myocarditis noninvasively. Due to its tissue characterization capability, cardiac magnetic resonance (CMR) has provided new insights into myocarditis-related injuries [8]. Tissue necrosis can be demonstrated with high spatial resolution, allowing differentiation of myocarditis from coronary syndromes [9]. A multisequential approach has been shown to be promising for the detection of myocarditis [10], and recently a consensus document on the CMR diagnosis of myocarditis has been published [11]. This multisequential approach is based on a combination of techniques; water sensitive $\mathrm{T} 2$ weighted imaging for the detection of myocardial tissue oedema, and early and late $\mathrm{T} 1$ weighted imaging after the injection of contrast agents such as gadolinium-diethylenetriaminepentaacetic acid (capillary leakage phase and presence of irreversibly injured myocardium). If two or more of the three tissue-based criteria are positive, myocardial inflammation can be predicted or ruled out with a diagnostic accuracy of $78 \%$ [11].

Myocarditis may become chronic due to viral persistence or autoimmune evolution. In this setting CMR may represent a useful non-invasive tool, and even guide the execution of EMB when indicated. In fact, the diagnosis of chronic myocarditis may have prognostic and therapeutic implications [12]. However, CMR data on chronic myocarditis are sparse and conflicting. Some investigators have shown that myocardial oedema as assessed by T2-weighted imaging is a reversible acute-phase injury [12], while others have shown oedema in a substantial fraction of patients with chronic myocarditis [10].

By adapting a comprehensive CMR approach we have previously shown the ability of early CMR to detect different tissue injuries in patients with suspected ST-elevation myocardial infarction (STEMI) and normal coronary arteries [13]. In the current report we adapt this CMR approach on suspected myocarditis patients and provide both the acute and follow-up data, and moreover, present the ability of CMR to visualize myocardial injuries over the course of the disease. A particular interest is shown in patients with ST-elevation vs. patients with no ST-elevation on admission.

\section{Materials and methods}

We studied two groups of patients who all presented with acute cardiac symptoms. One group of 26 patients presented with clinical suspicion of acute myocarditis and was included retrospectively. The second group of 16 patients mimicking myocardial infarction (MI) with acute ST-elevation was included prospectively (described in a previous publication [13]). All patients were admitted for the past 36 months and all were studied twice, within the first week and $12 \pm 9$ months later (finalized in March 2010). For inclusion, the patients had to fulfil each of the following clinical criteria: 1 . New onset of or persisting symptoms (chest pain and/or dyspnoe); 2. Evidence of recent or ongoing myocardial injury through elevation of troponin $\mathrm{T} ; 3$. Exclusion of coronary artery disease by coronary angiography, except for patients $<30$ years of age with a low pre-test probability for coronary artery disease [11]. Exclusion criteria were also previous MI $(n=3)$, takotsubo cardiomyopathy (transient left ventricular ballooning and no late gadolinium enhancement (LGE); $n=5$ ), and known contraindications to CMR (claustrophobia; $n=3$ ).

Based on these inclusion and exclusion criteria were 26 out of 58 patients presenting with suspicion of acute myocarditis eligible to participate in the study. Thirty-two patients showed no troponin $\mathrm{T}$ elevation and were excluded. Among these were 22 patients with a negative CMR and all were discharged with no final diagnosis. Ten patients showed findings on CMR (discharge diagnosis); focal subendocardial LGE (previous MI; $n=3$ ), reduced ejection fraction (EF) and increased end-diastolic volume (EDV) (dilated cardiomyopathy (DCM); $n=2$ ), focal pericardial gadolinium enhancement and effusion (pericarditis; $n=2$ ), increased myocardial thickness and mass (hypertrophic cardiomyopathy 
$(\mathrm{HCM}) ; n=1$ ), patchy midwall LGE (cardiac sarcoidosis; $n=1$ ), and diffuse subendocardial LGE (cardiac amyloidosis; $n=1$ ).

In the group mimicking MI were 16 out of 49 patients eligible to participate in the study based on these inclusion and exclusion criteria (excluded patients are previously described [13]). All participants gave informed written consent including retrospective analysis of data, and the study was approved by the Regional Ethics Committee and Review Board of our institution.

Coronary angiography was performed in 31 patients to exclude the presence of coronary artery disease $(<30 \%$ diameter stenosis $)$. Creatine kinase $(\mathrm{CK})$ and creatine kinase-myocardial band (CK-MB) was measured variably in both groups. C-reactive protein (CRP) and troponin $\mathrm{T}$ were measured on admission in all patients. ST-segment elevation was defined as J-point elevation $>1 \mathrm{~mm}$ in leads II, III, aVF, I, aVL, and/or $>2 \mathrm{~mm}$ in leads V1-6, with a total of minimum $2 \mathrm{~mm}$ in the inferior leads, and/or $4 \mathrm{~mm}$ in the anterior leads. All patients were asked for a follow-up CMR, preferably within 6 months.

\section{CMR imaging protocol}

The CMR studies were performed on a 1.5-Tesla unit (Intera CV, Philips Medical Systems, Best, The Netherlands). A standard five-channel phased-array cardiac coil was used with a signal correction shimming technique. For functional analysis ECGgated steady state free precession (SSFP) cine images of true anatomical axes of the heart were acquired (TE $1.9 \mathrm{~ms}$, TR $3.8 \mathrm{~ms}$, flip angle $60^{\circ}$ ). Then a breath hold, black-blood, T2 weighted triple inversionrecovery (IR) sequence (repetition time (TR) $2 \times R$ to $\mathrm{R}$ interval, echo time (TE) $80 \mathrm{~ms}$, inversion time (TI) $180 \mathrm{~ms}$ ) in short-axis covering the left ventricle (LV) (slice thickness $10 \mathrm{~mm}$, flip angle $180^{\circ}$, pixel size $0.96 \times 0.96 \mathrm{~mm}$ ) was used. Thereafter a short axis T1 weighted gradient echo IR sequence (TR $1 \times \mathrm{R}$ to $\mathrm{R}$ interval, TE $20 \mathrm{~ms}$, slice thickness $10 \mathrm{~mm}$ ) both before and after (without any change of parameters in between) an i.v. bolus of $0.15 \mathrm{mmol} / \mathrm{kg}$ gadolinium-diethylenetriaminepentaacetate (GdDTPA, Magnevist, Bayer Schering Pharma, Berlin, Germany) using an automated injector (Medrad, Indianola, USA) was introduced. The post-contrast measurements started immediately after injection and lasted for 3-4 min, thus, the images reflect gadolinium enhancement at a mean of 2 min (gRE). Finally, a breath-hold IR sequence (TR $6.1 \mathrm{~ms}$, TE $3.0 \mathrm{~ms}$, slice thickness $10 \mathrm{~mm}$ ) was applied after a delay of 15 min (LGE) in short- and long-axis covering the LV. TI were adjusted to null normal myocardium using the Look-Locker sequence.

\section{CMR analysis}

Off-line analyses were performed on a dedicated workstation (ViewForum release 5.2, Philips Medical Systems, Best, The Netherlands). Ventricular volumes and function were measured for both ventricles using standard techniques. The endocardial and epicardial borders were drawn manually on each dynamic image. The LVEF, EDV, systolic wall thickening (SWT), and myocardial mass were calculated from the short axis views. Two experienced observers analyzed all CMR images.

Visualization of myocardial oedema was performed using the T2-weighted sequence by measuring the ratio of global myocardial signal intensity (SI) to skeletal muscle (drawn in the same slice). The T2-ratio was calculated as SI myocardium/SI skeletal muscle, and a $\mathrm{T} 2$ ratio of 2.0 was used as cut-off value. The gRE sequence was measured in a similar way, and regions of interest were manually drawn and copied from the pre-contrast to the post-contrast images. The gRE was calculated as previously described [14] and a cut-off value of 4.0 was used. The LGE sequence was analyzed segment by segment (17-segment model as proposed by the American Heart Association) for the presence, number, and transmurality of LGE areas. The spatial extent of enhancement was calculated $(>2$ standard deviations (SD) compared to a normal segment) and expressed in grams (LV mass; calculated from the product of enhanced volume and specific gravity of myocardium [1.05 g/ml]). The CMR images were also analysed for the presence of concomitant pericardial effusion and/or enhancement of the pericardial layers. The diagnosis of myocarditis was made when two or more out of three sequences (T2, gRE, LGE) were abnormal. A control group, which has been presented earlier [13], was used for the validation of CMR sequences and determination of imaging specific cut-off values. We also used the validation process to optimize homogeneity with use of the phased array coil and signal intensity correction software. 
Statistical analysis

Each categorical variable is expressed as number and percentage of patients. Demographical and clinical data are presented as mean with SD or proportions. Baseline characteristics were compared using an independent sample t-test or Mann-Whitney test as appropriate. A chi-square test for contingency tables was obtained to detect associations between categorical independent variables. When comparing continuous variables, a twosided Student's t test was used. Data were correlated using Spearman correlation coefficient and linear regression. $P$-values less than 0.05 were considered statistically significant, and $P$-values less than 0.001 highly significant. All statistical analysis was performed using GraphPad Prism for Macintosh, version 5.0 (GraphPad Software, La Jolla, CA, USA).

\section{Results}

Table 1 summarizes the characteristics of the study population. In total, 42 patients (39 males, three females; age $37 \pm 14$ years) were recruited. The main cardiac symptom was chest pain $(80 \%)$. Eleven patients (22 \pm 5 years) did not undergo coronary angiography.

Twelve patients showed coronary stenosis $<30 \%$ in at least one coronary artery segment, while 19 patients showed completely normal coronary arteries with no lumen irregularities. However, provocative tests for the exclusion of coronary spasm were not performed. ECG on admission showed nonspecific ST-T-changes in 19 patients, ST-elevation in 18

Table 1 Baseline characteristics

\begin{tabular}{ll}
\hline & $n=42$ \\
\hline Mean age (years) & $37 \pm 14$ \\
Male sex $[n(\%)]$ & $39(93)$ \\
Diabetes $[n(\%)]$ & $5(12)$ \\
Hypertension $[n(\%)]$ & $6(14)$ \\
Smoking $[n(\%)]$ & $13(31)$ \\
Infection or flu $[n(\%)]$ & $26(62)$ \\
Mean troponin T $(\mu \mathrm{g} / \mathrm{l})$ & $0.93 \pm 0.95$ \\
Mean CK $(\mu \mathrm{g} / \mathrm{l})$ & $48 \pm 63$ \\
Mean CK-MB $(\mu \mathrm{g} / \mathrm{l})$ & $517 \pm 364$ \\
Mean CRP $(\mathrm{mg} / \mathrm{l})$ & $56 \pm 78$ \\
\hline
\end{tabular}

patients (anterior leads $6.5 \pm 3.6 \mathrm{~mm}$, inferior leads $1.9 \pm 2.2 \mathrm{~mm}$ ), a normal ECG in four patients, and a right bundle branch block in one patient. In addition two of these patients presented with AV-block and two patients with incomplete intraventricular block. The average time between the initial symptoms and admission was $0.8 \pm 1.2$ days, and between admission and the first CMR $2.9 \pm 2.4$ days. There was a mild to moderate elevation in troponin $\mathrm{T}$ $(0.93 \pm 0.90 \mu \mathrm{g} / \mathrm{l})$ for all patients, although two patients showed a borderline value of $0.02 \mu \mathrm{g} / \mathrm{l}$. CRP $(56 \pm 78 \mathrm{mg} / \mathrm{l})$ showed increased values in 23 patients $(55 \%)$, and 26 patients $(62 \%)$ reported a recent history of infection or flu-like symptoms before the onset of cardiac symptoms. Also, 2 patients with diabetes had an ongoing ketoacidosis on admission. There was a moderate increase in CK $(48 \pm 63 \mu \mathrm{g} / \mathrm{l})$ and CK-MB $(517 \pm 364 \mu \mathrm{g} / \mathrm{l})$.

Acute phase CMR

Three T1 weighted sequences early after i.v. GdDTPA (gRE) were of suboptimal quality, otherwise all CMR examinations were of diagnostic quality and results are summarized in Table 2 . The $\mathrm{T} 2$ ratio was elevated in 24 patients $(57 \%)$, gRE in 13 patients $(31 \%)$, and LGE was present in 27 patients $(64 \%)$. In 32 patients $(76 \%)$ were any two (or more) out of three sequences (T2, gRE, LGE) abnormal (Fig. 1). Compared to controls, the $\mathrm{T} 2$ ratio was significantly elevated ( $2.04 \pm 0.32$ vs. $1.68 \pm 0.19, P<0.001)$, as was the gRE $(4.07 \pm 1.63$ vs. $2.95 \pm 0.82 ; P<$ $0.05)$, and the myocardial mass $(150 \pm 28$ vs. $126 \pm 36 \mathrm{~g} ; \quad P<0.05)$. There was no significant change of $\mathrm{EF}(57.4 \pm 11.9$ vs. $62.8 \pm 7.3 \%$; ns), EDV (158 \pm 46 vs. $160 \pm 42 \mathrm{ml}$; ns), or SWT (61 \pm 18 vs. $75 \pm 20 \%$; ns) compared to controls. LGE mass was $9.2 \pm 8.6 \mathrm{~g}$ and LGE were mainly seen in the infero-lateral segments $(4,5,10,11 ; 86 \%)$, and all LGE was seen in the mid or subepicardial regions. Pericardial effusion was seen in seven patients $(17 \%)$ at the acute event.

\section{Prognosis and follow-up CMR}

Each of the 42 patients survived the acute event. Clinical follow-up was completed for all patients and revealed no major adverse cardiac events. 36 patients (86\%) returned for the follow-up CMR $12 \pm 9$ months 
Table 2 CMR results at baseline and follow-up

$n s$ non significant

\begin{tabular}{llll}
\hline & Baseline $(n=42)$ & Follow-up $(n=36)$ & $P$ value \\
\hline LV EF (\%) & $57.4 \pm 11.9$ & $61.4 \pm 7.6$ & $<0.05$ \\
LV EDV (ml) & $158 \pm 46$ & $143 \pm 34$ & $\mathrm{~ns}$ \\
LV mass (g) & $150 \pm 28$ & $134 \pm 24$ & $<0.05$ \\
LV SWT (\%) & $61 \pm 18$ & $72 \pm 26$ & $<0.05$ \\
Elevated T2 ratio [n (\%)] & $24(57)$ & $4(11)$ & $<0.001$ \\
Mean T2 ratio & $2.04 \pm 0.32$ & $1.70 \pm 0.28$ & $<0.001$ \\
Elevated gRE [n $(\%)]$ & $13(31)$ & $6(17)$ & $<0.05$ \\
Mean gRE & $4.07 \pm 1.63$ & $3.11 \pm 1.22$ & $<0.05$ \\
LGE [n $(\%)]$ & $27(64)$ & $10(28)$ & $<0.001$ \\
Any 2 out of 3 sequences & & & $<0.001$ \\
Abnormal $(\%)$ & $32(76)$ & $8(22)$ & $\mathrm{ns}$ \\
Mean LGE mass $(\mathrm{g})$ & $9.2 \pm 8.6$ & $5.5 \pm 2.2$ & $\mathrm{~ns}$ \\
Pericardial effusion $[n(\%)]$ & $7(17)$ & $4(11)$ & \\
\hline
\end{tabular}
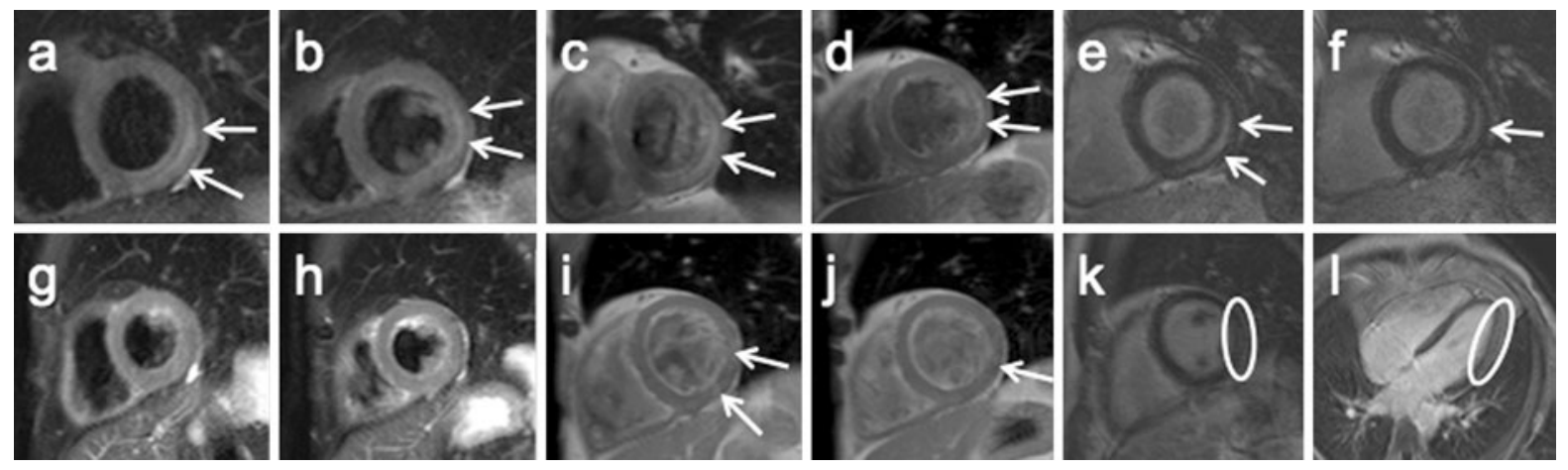

Fig. 1 Patient with myocarditis (a-1). CMR demonstrates T2weighted $(\mathbf{a}, \mathbf{b}, \mathbf{g}, \mathbf{h}), \operatorname{gRE}(\mathbf{c}, \mathbf{d}, \mathbf{i}, \mathbf{j})$, and LGE $(\mathbf{e}, \mathbf{f}, \mathbf{k}, \mathbf{l})$ during the acute phase (top) and follow-up (bottom). During the acute phase, lateral intramural signal elevation is seen in all images

after presentation. Three patients declined the followup CMR and three patients were unable to perform the CMR due to an implanted cardioverter-defibrillator (ICD; $n=2)$ or a pacemaker (PM; $n=1)$. Another patient had the follow-up CMR performed before the PM implantation. There was a significant decrease in myocardial oedema (T2 ratio; $2.04 \pm 0.32$ vs. $1.70 \pm 0.28 ; \quad P<0.001), \quad$ gRE $\quad(4.07 \pm 1.63$ vs. $3.11 \pm 1.22 ; P<0.05$ ) (Figs. 1 and 2 ), and myocardial mass $(150 \pm 28 \mathrm{~g}$ vs. $137 \pm 24 ; P<0.05)$, as well as an increase of LVEF $(57.4 \pm 11.9 \%$ vs. $61.4 \pm 7.6 ; \quad P<0.05$ ) (Fig. 2) and systolic wall thickening (SWT; $61 \pm 18 \%$ vs. $72 \pm 26 \% ; P<$ 0.05). No significant change was seen in EDV (158 \pm 46 vs. $143 \pm 34$; ns $)$ or LGE mass $(9.2 \pm 8.6$ vs. $5.5 \pm$ $2.2 \mathrm{~g}$; ns) compared to the acute phase. The LGE (white arrows). At follow-up, the T2 changes are not seen, but gRE and LGE lesions persisted (spotty appearance; white arrows/circles)

persisted in 10 patients and pericardial effusion in 4 patients. Totally 8 patients had two or more abnormal sequences (T2, gRE, LGE) also at follow-up. There was no relation between the degree of change in CMR parameters of myocardial injury (delta-T2, delta-gRE, and presence of LGE) and the change in functional parameters over the course of myocarditis. Also, the extent of LGE did not correlate with any of the functional parameters. One patient was readmitted with acute myocarditis within the observation period.

ST-elevation vs. non-ST-elevation

There was a significant difference in time to admission and CMR between the groups. Time from the initial symptoms to admission was significant lower 

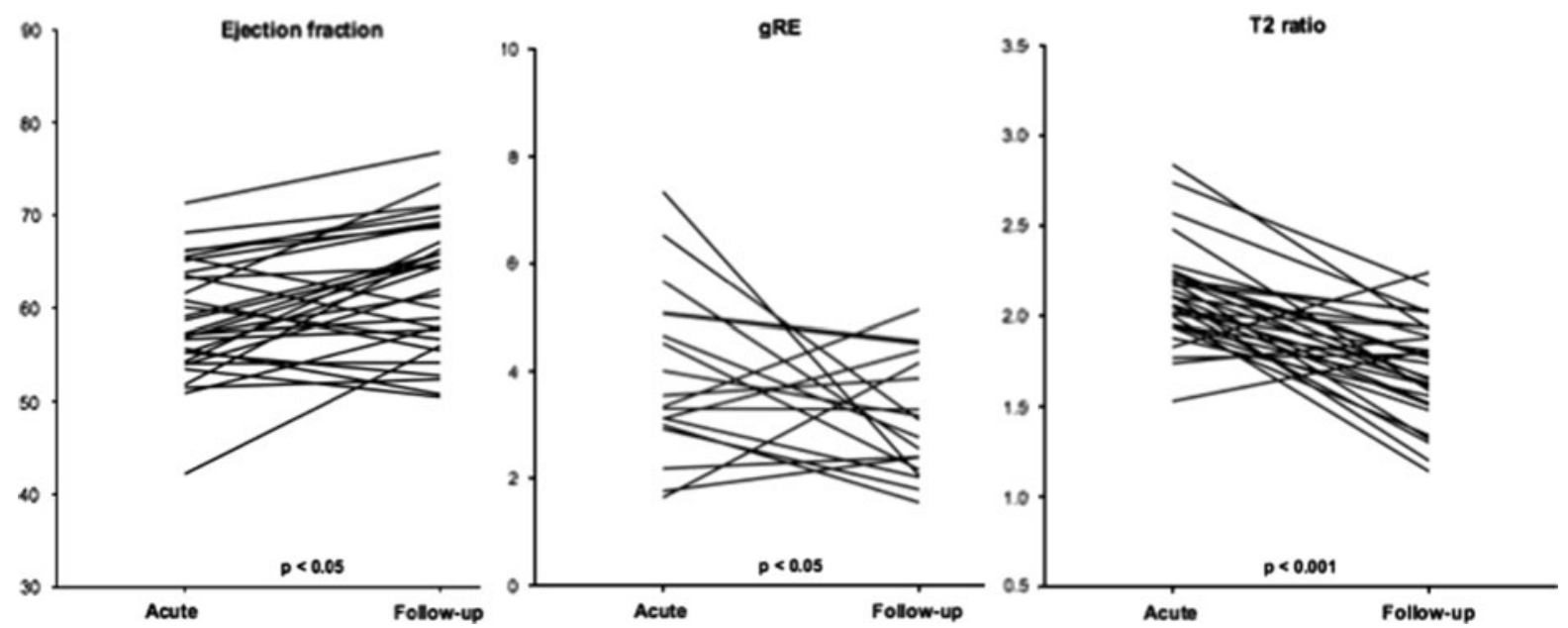

Fig. 2 Line graphs demonstrate the changes in ejection fraction (EF), gRE, and T2 ratios over the course of myocarditis. The increased $\mathrm{EF}(P<0.05)$ is paralleled by a normalization of $\operatorname{gRE}(P<0.05)$ and $\mathrm{T} 2$ ratio $(P<0.001)$

in the group with ST-elevation compared to the non-ST-elevation group $(4 \pm 2$ vs. $29 \pm 36 \mathrm{~h}$; $P=0.002)$, as was the time from admission to CMR $(0.9 \pm 0.6$ vs. $4.1 \pm 2.2$ days; $P<0.001)$. For the ST-elevation group on admission there was a trend toward decreased LVEF (55.9 \pm 7.3 vs. $59.9 \pm 10.8 \% ; P=0.13)$ and $\operatorname{gRE}(3.82 \pm 1.77 \mathrm{vs}$. $4.27 \pm 1.63 ; P=0.24)$, as well as increased $\mathrm{T} 2$ ratio $(2.09 \pm 0.10 \pm 2.01 \pm 0.41 ; P=0.15)$ compared to the non-ST-elevation group. At follow-up there was a similar trend toward decreased LVEF (58.7 \pm 8.1 vs. $63.3 \pm 6.6 ; P=0.07)$ and $\operatorname{gRE}(2.65 \pm 0.95$ vs. $3.43 \pm 1.31 ; P=0.09$ ) for the ST-elevation group. The differences did not reach statistical significance, probably in part due to a relatively small sample size. However, the LGE mass was significantly increased for the ST-elevation group both on admission $(12.3 \pm 11.9$ vs. $7.1 \pm 4.6 \mathrm{~g} ; \quad P<0.05)$ and at follow-up $(6.8 \pm 2.7$ vs. $4.5 \pm 1.3 \mathrm{~g} ; \quad P<0.05)$ compared to the non-ST-elevation group.

\section{Differential diagnosis}

DCM was present in three patients (Fig. 3). One patient had a heart arrest shortly after admission (EDV $254 \mathrm{ml}$, EF 52\%, no LGE), and one patient presented with a severely reduced EF of $15 \%$ (EDV $202 \mathrm{ml}$, no LGE). The third patient had a moderately reduced EF of $31 \%$ (EDV $348 \mathrm{ml}$, gRE 8.1 and LGE in lateral segments $5,6,11,15,16)$. Two of the DCM patients had an ICD successfully implanted. Two other patients had an AV-block on admission and one was successfully treated for a heart arrest (no LGE). The second patient had an AV-block grade 2 that subsequently became grade 3 (gRE 4.66 and LGE in segments 2 and 3). Both these patients successfully had a PM implanted. Additionally, four patients presented with a minimally raised troponin $\mathrm{T}(0.18 \pm$ $0.14 \mu \mathrm{g} / \mathrm{l})$ as the only finding, since all CMR parameters were normal and no LGE was demonstrated in the acute phase or at follow-up.

\section{Discussion}

CMR has emerged as a valuable tool in the noninvasive diagnosis of myocarditis since the first case series with T2-weigthed spin-echo sequences in 1991 [15] and gadolinium-enhanced T1-weighted images to serially image the natural history of acute myocarditis in 1998 [14]. A combination of techniques with the introduction of T2-weighted sequences and comparison of myocardial with skeletal muscle enhancement, have further improved the diagnostic accuracy [16]. A consensus report from 2009 suggested criteria providing evidence for or against myocardial inflammation based on CMR findings [11].

This suggested multisequential approach for suspected myocarditis has been performed in our institution since 2007. So far reports documenting 

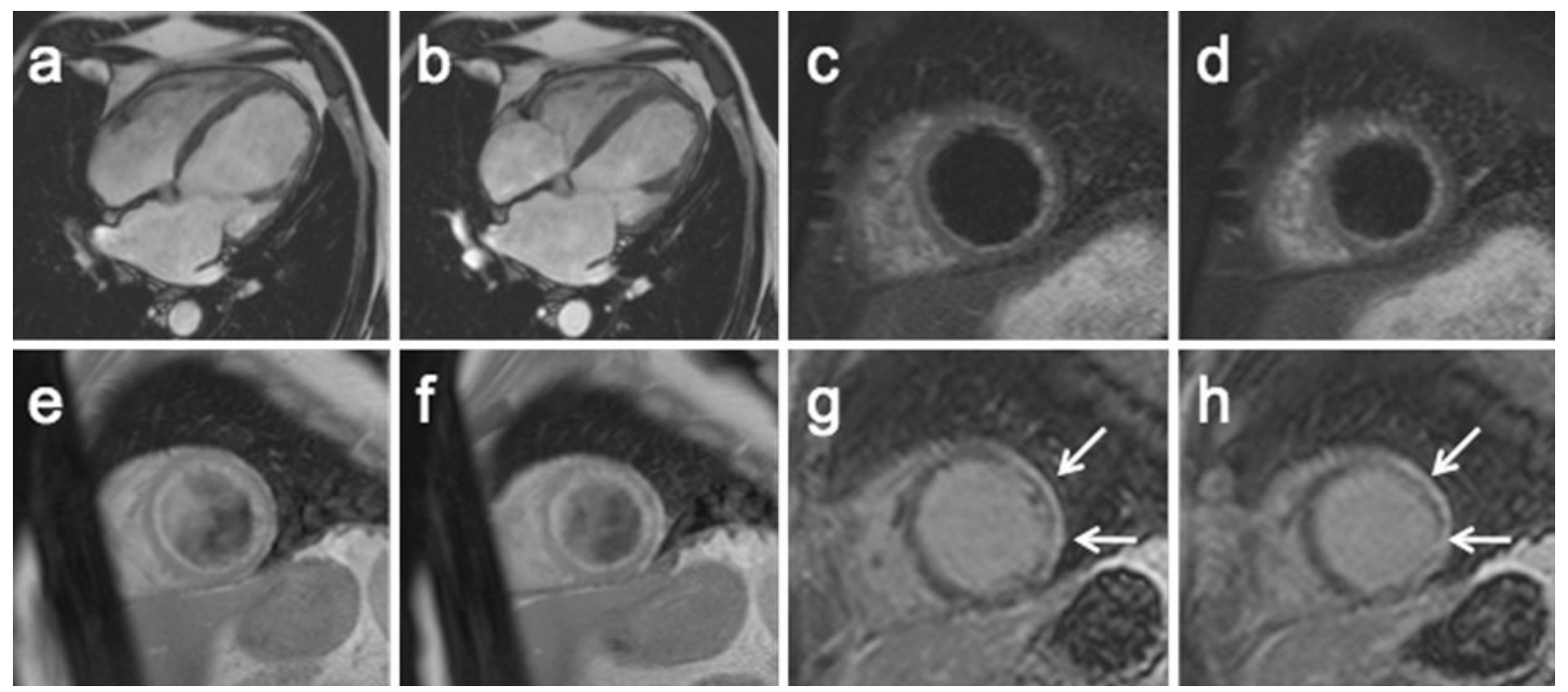

Fig. 3 CMR demonstrates a patient with DCM (a-h). EF is $19 \%$ and EDV $348 \mathrm{ml}$ (a; end-diastole, b; end-systole). The T2-weighted (c, d) and gRE (e, f) images are normal, but there

is lateral epicardial LGE ( $\mathrm{g}$, $\mathrm{h}$; white arrows). This patient had later an ICD implanted and no follow-up CMR was done

this CMR approach in suspected myocarditis are rare. We have in this report been able to show that this CMR approach is capable of monitoring myocardial injuries over the course of myocarditis. Also, this is the first study to show increased myocardial injuries for myocarditis patients with ST-elevation compared to non-ST-elevation. In addition, our study showed that a large group of patients (34\%) had markedly elevated troponin in the absence of LGE. Accordingly, CMR added information for the differential diagnosis, which is important because of different treatment strategies.

Myocardial tissue oedema [17] and increased capillary leakage [18] are the most likely pathological correlates to the $\mathrm{T} 2$ ratio and gRE. These injuries are reversible and transient features of the inflammatory response, and could thus explain our CMR findings. Our study showed that both T2 and gRE in a majority of patients decreased over the course of myocarditis, and that this reduction was associated with improvement in some LV function parameters. These findings are supported by the recently reported observations by Zagrosek [19]. It is possible that a complete recovery of myocarditis is associated with a reduction of the $\mathrm{T} 2$ ratio and $\mathrm{gRE}$ as in our study. Also, we have recently shown that $\mathrm{T} 2$ changes reflecting myocardial oedema are features of the acute but not of the chronic injury in patients with suspected STEMI and normal coronary arteries [13]. A recent

CMR study that also included EMB found that T2 changes are rare in chronic myocarditis [12]. The same findings have also been shown in myocardial infarction [20], lending further support to our current findings, and as such, CMR may offer a usable tool to monitor disease progression or regression.

The incidence of LGE in myocarditis is a controversial issue. LGE persisted over the course of myocarditis in $28 \%$ of our patients, which is a somewhat lower percentage compared to some reports [21], but higher compared to others [10]. The discrepancy may be related to differences in patient populations or study designs. Furthermore, the pattern of myocardial injury is influenced by the virus type [22]. We recently published a case report of the first H1N1 myocarditis (3 abnormal sequences) and a complete recovery after 3 months [23]. In fact, our findings showed that a majority of the patients had reversible myocardial injury, and that sensitivity of LGE will remain a controversial issue due to the fact that $34 \%$ of the patients had markedly elevated troponin in the absence of any LGE. A possible explanation is CMRs inability to demonstrate diffuse myocardial changes as possibly encountered in some myocarditis patients. This is an important reason to perform a comprehensive CMR approach in patients with suspected myocarditis. A meta analysis (from the consensus report) showed that if all sequences can be performed and 2 or more of the 3 tissue-based criteria 
are positive, myocardial inflammation can be predicted or ruled out with a diagnostic accuracy of $78 \%$; if only LGE imaging is performed, the diagnostic accuracy is reduced to $68 \%$ [11]. Also the presence of pericardial effusion as seen in 7 of our patients may provide additional evidence for myocarditis.

Other than expected, findings of LGE was not always associated with a focal increase in T2 signal or increased gRE, neither was there any significant correlation between these findings. It seems that the evolution pattern of myocardial oedema and capillary leakage might be different from LGE. Accordingly, at a particular "time window", only one or two of three parameters are detectable. Serial studies have shown that gRE needs a longer time delay after the onset of symptoms to become positive, and although there was a difference in time delay between our patients with or without ST-elevation, the time delay might not be long enough. The $\mathrm{T} 2$ abnormalities allow the detection of tissue oedema, a substantial and rapid feature of the acute inflammatory reaction in the myocardium. Possibly, at least in some patients, focal oedema marks a less severe form of injury that may not progress to actual necrosis. On the other hand, the differences in time delay might explain the significant difference that was seen in LGE mass between the groups, as an increased LGE mass for the ST-elevation group tended to show increased $\mathrm{T} 2$ ratio and gRE as well as decreased LVEF on admission.

Our patient population was fairly homogenous with predominantly young males and nearly normal LV function and dimensions, and a complete recovery was seen in a majority of our patients. This finding is in agreement with previous reports [19]. Mahrholdt recently observed an association between the pattern of LGE and LV remodelling in myocarditis [21]. However, approximately $50 \%$ of patients in that study presented with LV dilatation and impaired EF in which initial EDV emerged as an independent predictor of later LV dilatation. The link between myocarditis and the later development of DCM is well established [24]. Yet only a fraction of myocarditis patients progress to dilated DCM. Three of our patients presented with DCM, but only one showed LGE and increased gRE. It is not known whether myocarditis patients with positive CMR parameters may be more likely to develop DCM, but it seems intriguing to postulate. Also, whether myocarditis patients would be more liable to develop ventricular arrhythmias will be a future research focus.

\section{Limitations}

The lack of EMB is a limitation of our study. However, the sensitivity of EMB is limited [22], and it may have severe complications. Also, the majority of our patients were young with an uneventful recovery, and we avoided unnecessary risks for the patients. There is a theoretical possibility that some patients suffered from undetected coronary heart disease, such as a resolved thrombus, but the lack of scarring by CMR makes an ischemic injury unlikely. Both the body coil and the phased array coil have been used in the T2 and the gRE sequences in other studies. The use of the body coil and axial views may show somewhat lower noise levels, but in our experience optimized use of the phased array coil with effective signal intensity correction software allows sufficient signal homogeneity. Finally, the time to follow-up was variable, reflecting the retrospective nature of this analysis.

\section{Conclusion}

In conclusion, this CMR study provides further insights into the pathophysiological mechanisms in myocarditis, and demonstrates that a comprehensive CMR approach is able to monitor myocardial tissue injuries over the course of the disease. Both acute and chronic injuries tend to be more pronounced in patients with initial ST-elevation. Additionally, CMR has incremental value for differential diagnoses and therapeutic decision-making, and therefore recommended in all patients with acute cardiac symptoms, elevated cardiac biomarkers, and unobstructed coronary arteries.

Acknowledgments The authors are grateful for the excellent assistance of Vigdis Rosseland, Marianne Nesheim, and Grethe Hansen.

Open Access This article is distributed under the terms of the Creative Commons Attribution Noncommercial License which permits any noncommercial use, distribution, and reproduction in any medium, provided the original author(s) and source are credited. 
Conflict of interest None.

\section{References}

1. Feldman AM, McNamara D (2000) Myocarditis. N Engl J Med 343:1388-1398

2. Doolan A, Langlois N, Semsarian C (2004) Causes of sudden cardiac death in young Australians. Med J Aust 180:110-112

3. Kawai C (1999) From myocarditis to cardiomyopathy: mechanisms of inflammation and cell death: learning from the past for the future. Circulation 99:1091-1100

4. Calabrese F, Basso C, Carturan E, Valente M, Thiene G (2006) Arrhythmogenic right ventricular cardiomyopathy/ dysplasia: is there a role for viruses? Cardiovasc Pathol 15:11-17

5. Masoudi FA, Magid DJ, Vinson DR, Tricomi AJ, Lyons EE, Crounse L, Ho PM, Peterson PN, Rumsfeld JS (2006) Implications of the failure to identify high-risk electrocardiogram findings for the quality of care of patients with acute myocardial infarction: results of the Emergency Department Quality in Myocardial Infarction (EDQMI) study. Circulation 114:1565-1571

6. Liu PP, Yan AT (2005) Cardiovascular magnetic resonance for the diagnosis of acute myocarditis: prospects for detecting myocardial inflammation. J Am Coll Cardiol 45:1823-1825

7. Cooper LT, Baughman KL, Feldman AM, Frustaci A, Jessup M, Kuhl U, Levine GN, Narula J, Starling RC, Towbin J, Virmani R (2007) The role of endomyocardial biopsy in the management of cardiovascular disease: a scientific statement from the American Heart Association, the American College of Cardiology, and the European Society of Cardiology. Circulation 116:2216-2233

8. Skouri HN, Dec GW, Friedrich MG, Cooper LT (2006) Noninvasive imaging in myocarditis. J Am Coll Cardiol 48:2085-2093

9. Laissy JP, Hyafil F, Feldman LJ, Juliard JM, SchoumanClaeys E, Steg PG, Faraggi M (2005) Differentiating acute myocardial infarction from myocarditis: diagnostic value of early- and delayed-perfusion cardiac MR imaging. Radiology 237:75-82

10. Gutberlet M, Spors B, Thoma T, Bertram H, Denecke T, Felix R, Noutsias M, Schultheiss HP, Kuhl U (2008) Suspected chronic myocarditis at cardiac MR: diagnostic accuracy and association with immunohistologically detected inflammation and viral persistence. Radiology 246:401-409

11. Friedrich MG, Sechtem U, Schulz-Menger J, Holmvang G, Alakija P, Cooper LT, White JA, Abdel-Aty H, Gutberlet M, Prasad S, Aletras A, Laissy JP, Paterson I, Filipchuk NG, Kumar A, Pauschinger M, Liu P (2009) Cardiovascular magnetic resonance in myocarditis: a JACC White paper. J Am Coll Cardiol 53:1475-1487

12. De Cobelli F, Pieroni M, Esposito A, Chimenti C, Belloni E, Mellone R, Canu T, Perseghin G, Gaudio C, Maseri A,
Frustaci A, Del Maschio A (2006) Delayed gadoliniumenhanced cardiac magnetic resonance in patients with chronic myocarditis presenting with heart failure or recurrent arrhythmias. J Am Coll Cardiol 47:1649-1654

13. Stensaeth KH, Fossum E, Hoffmann P, Mangschau A, Klow NE (2010) Clinical characteristics and role of early cardiac magnetic resonance imaging in patients with suspected ST-elevation myocardial infarction and normal coronary arteries. Int J Cardiovasc Imaging. doi:10.1007/ s10554-010-9671-7

14. Friedrich MG, Strohm O, Schulz-Menger J, Marciniak H, Luft FC, Dietz R (1998) Contrast media-enhanced magnetic resonance imaging visualizes myocardial changes in the course of viral myocarditis. Circulation 97:1802-1809

15. Gagliardi MG, Bevilacqua M, Di Renzi P, Picardo S, Passariello R, Marcelletti C (1991) Usefulness of magnetic resonance imaging for diagnosis of acute myocarditis in infants and children, and comparison with endomyocardial biopsy. Am J Cardiol 68:1089-1091

16. Laissy JP, Messin B, Varenne O, Iung B, Karila-Cohen D, Schouman-Claeys E, Steg PG (2002) MRI of acute myocarditis: a comprehensive approach based on various imaging sequences. Chest 122:1638-1648

17. Boxt LM, Hsu D, Katz J, Detweiler P, Mclaughlin S, Kolb TJ, Spotnitz HM (1993) Estimation of myocardial water content using transverse relaxation time from dual spinecho magnetic resonance imaging. Magn Reson Imaging 11:375-383

18. Swanson SD, Dou C, Miller DL (2006) Magnetic resonance imaging of microvascular leakage induced by myocardial contrast echocardiography in rats. Magn Reson Imaging 24:603-609

19. Zagrosek A, Abdel-Aty H, Boye P, Wassmuth R, Messroghli D, Utz W, Rudolph A, Bohl S, Dietz R, SchulzMenger J (2009) Cardiac magnetic resonance monitors reversible and irreversible myocardial injury in myocarditis. JACC Cardiovasc Imaging 2:131-138

20. Abdel-Aty H, Zagrosek A, Schulz-Menger J, Taylor AJ, Messroghli D, Kumar A, Gross M, Dietz R, Friedrich MG (2004) Delayed enhancement and T2-weighted cardiovascular magnetic resonance imaging differentiate acute from chronic myocardial infarction. Circulation 109:2411-2416

21. Mahrholdt H, Wagner A, Deluigi CC, Kispert E, Hager S, Meinhardt G, Vogelsberg H, Fritz P, Dippon J, Bock CT, Klingel K, Kandolf R, Sechtem U (2006) Presentation, patterns of myocardial damage, and clinical course of viral myocarditis. Circulation 114:1581-1590

22. Mahrholdt H, Goedecke C, Wagner A, Meinhardt G, Athanasiadis A, Vogelsberg H, Fritz P, Klingel K, Kandolf R, Sechtem U (2004) Cardiovascular magnetic resonance assessment of human myocarditis: a comparison to histology and molecular pathology. Circulation 109:1250-1258

23. Weiss TW, Stensaeth KH, Eritsland J (2010) Myocarditis in a juvenile patient with influenza A virus infection. Eur Heart J 31:277

24. Sole MJ, Liu P (1993) Viral myocarditis: a paradigm for understanding the pathogenesis and treatment of dilated cardiomyopathy. J Am Coll Cardiol 22:99A-105A 